\title{
YEAR IN REVIEW: \\ COMMUNICABLE DISEASE SURVEILLANCE, 2000
}

In this issue, we review the trends in reports of notifiable diseases received by the NSW Department of Health for 2000. Readers interested in the details of specific diseases should review Tables 1 to 4 for notifications of disease reports by year, month, area of residence, and age group and sex.

\section{TRENDS}

Among the 30,020 people with medical conditions notified by doctors, hospital staff, and laboratories for 2000 :

\section{Conditions most frequently reported}

- hepatitis C (7,513 cases [116/100,000 population], with the highest crude rates among residents who live within the Western Sydney, Mid Western NSW, and Central Sydney area health services);

- hepatitis B (4,008 cases [62/100,000], with the highest crude rates among residents who live within the South West Sydney, Central Sydney and the Far Western area health services);

- pertussis $(3,682$ cases [57/100,000], with the highest crude rates among residents who live within the Hunter, Mid Western and Macquarie area health services);

- chlamydia (3,464 cases [54/100,000], with the highest crude rates among residents who live within the Far Western, South Eastern Sydney and Central Sydney area health services);

Conditions with the most important declines in notifications over previous years

- AIDS (102 cases, down from a peak of 533 cases in 1994) and HIV infection (367 cases, steadily declining since the mid-1980s): both declines are most likely due to effective new therapies for HIV infection;

- hepatitis A (195 cases, the lowest number since laboratory reporting for hepatitis A began in 1991): possibly due to relatively high levels of immunity within the community through past infection or immunisation, or possibly due to improved hygiene;

- Q fever (down to 128 cases from a peak of 404 cases in 1993): possibly due to higher immunisation rates among abattoir workers;

- $\quad$ salmonellosis (1,387 cases, down from a peak of 1,811 cases in 1998);

Conditions with the most important increases over previous years

- pertussis (3,682 cases, up from 1,414 cases in 1999): due to an epidemic mainly in older children and adults;

- chlamydia (3,464 cases, up from 2,438 cases in 1999): perhaps related to improved reporting by laboratories, and ongoing risk activities among sexually active men and women;
- rubella (190 laboratory confirmed cases, up from 46 cases in 1999): largely related to an outbreak among non-immunised adult males;

\section{Conditions least frequently reported}

There were no reported cases of botulism, chancroid, cholera, diphtheria, lymphogranuloma venereum (LGV), donovanosis, plague, polio, rabies, typhus, viral haemorrhagic fevers, or yellow fever.

\section{OUTBREAKS}

Several important disease outbreaks were reported in 2000 in NSW. These include:

- an outbreak of shigellosis among gay men in inner Sydney; ${ }^{1}$

- a large outbreak of pertussis affecting mainly older children and adults statewide; ${ }^{2}$

- an outbreak of influenza among persons aboard a South Pacific cruise ship; ${ }^{3}$

- two clusters of acute post streptococcal glomerular nephritis among children in rural and urban NSW. ${ }^{4}$

\section{POLICY DEVELOPMENT}

In 2000, the NSW Department of Health's Communicable Diseases Surveillance and Control Unit (CDSCU) led the development of several important strategies for the control and prevention of communicable diseases in NSW, including:

- surveillance and control for communicable diseases that occurred during the period of the Olympic Games and the Paralympic Games, ${ }^{5}$

- a revised Notifiable Diseases Manual to assist public health investigations;

- new surveillance systems for anthrax, influenza, invasive pneumococcal diseases, lyssavirus infection, psittocosis and shigellosis, which became notifiable conditions in NSW

- development of communicable disease control priorities for NSW; 6

- development of policy on health care worker protection;

- development of policy of the prevention of tuberculosis in health care workers; ${ }^{7}$

- development of a new intranet page on Communicable Disease Control for staff of NSW Health at internal.health.nsw.gov.au/public-health/cdscu;

- development and installation of a new version of Notifiable Diseases Database in public health units;

- release of the Chest Clinic Surveillance System database Version 2. 


\section{TRAINING AND INFORMATION}

In 2000, the CDSCU facilitated training for public health professionals in communicable disease control and developed new information resources, including:

- the NSW Communicable Disease Control Workshop for public health units, laboratories and trainees;

- the NSW Tuberculosis Conference;

- Bug Breakfast seminars for public health professionals in NSW;

- supervision of two trainees in the NSW Public Health Officer Training Program;

- teaching students of public health and medicine at the Universities of Sydney and New South Wales;

- new fact sheets on headlice, chickenpox, hepatitis $\mathrm{C}$, parvovirus B19 and fifth disease, meningococcal disease, Ross River virus infection, tuberculosis, hepatitis A, whooping cough and influenza (www.health.nsw.gov.au);

- epidemiological reviews on meningococcal disease, tuberculosis, arboviruses, and HIV and AIDS (www.health.gov.au/public-health/phb/phb.html).

\section{PROGRESS ON PRIORITY AREAS}

In 2000, the CDSCU identified seven priority areas for development in NSW. ${ }^{6}$ Here we report on progress on these across the state:

- eliminate the transmission of measles: 20 laboratoryconfirmed and 12 other cases in 2000. Seven measles cases were thought to have been acquired overseas, including three in overseas visitors to Australia;

- eliminate congenital rubella: no cases in 2000;

- eliminate congenital syphilis: one case in 2000;

- monitor risk factors for new hepatitis $C$ infections: 138 acute cases reported. Risk factor data on acute cases have been investigated by many public health units and will be reported in a future issue of the NSW Public Health Bulletin;

- better understand risk factors for invasive pneumococcal disease (IPD): IPD became a notifiable disease at the end of 2000;

- minimise the incidence and management of multi-drug resistant tuberculosis (MDR-TB): six cases were identified in 2000. The management of all cases were reviewed by an expert panel;

- minimise the risk of communicable disease infections related to the Olympic and Paralympic Games in Sydney: a comprehensive surveillance system was developed. ${ }^{7}$ No communicable disease outbreaks were identified related to the Olympic or Paralympic Games.

\section{INITIATIVES FOR 2001}

To strengthen communicable disease control activities in 2001, the following initiatives were planned:

- development of protocols for the response of public health units to reports of anthrax, psittacosis, invasive pneumococcal disease, influenza and shigellosis;

- a training workshop for public health workers in outbreak investigation and management;

- streamlining of HIV data collection, analysis and reporting;

- analysis of risk factors among new cases of hepatitis C;

- release of the Web page for communicable disease control.

\section{A BIG THANK YOU}

Disease control and prevention depends on effective surveillance of communicable diseases in the community. The CDSCU would like to thank all those general and specialist medical practices, laboratories, hospitals, schools, childcare centres, and others, who have notified diseases of public health significance to their local public health units for investigation and control.

\section{REFERENCES}

1 NSW Department of Health. Shigellosis outbreak among inner-Sydney men. NSW Public Health Bulletin 2000; 11 : 158.

2 NSW Department of Health. Pertussis on the rise. NSW Public Health Bulletin 2000; 11: 174.

3 NSW Department of Health. Outbreak of influenza-like illness on board a cruise ship. NSW Public Health Bulletin 2000; 11 : 194-195.

4 Muscatello DJ, O'Grady KA, Neville K, McAnulty J. Acute poststreptococcal glomerulonephritis: Public health implications of recent clusters in NSW and epidemiology of hospital admissions. Epidemiol Infect 2001; 126: 365-372.

5 Thackway SV, Delpech VC, Jorm LR, McAnulty JM, Visotina M. Monitoring acute diseases during the Sydney 2000 Olympic and Paralympic Games. Med J Aust 2000; 173: 318321.

6 NSW Department of Health. Draft priorities for communicable disease control in NSW, 2000. NSW Public Health Bulletin 2000; 11: 84-86.

7 NSW Department of Health. Health care worker tuberculosis screening and protection policy. Circular 2001/71; 1 August 2001. it 
TABLE 1

DISEASE NOTIFICATIONS BYYEAR OF ONSET, NSW, 1991 TO 2000

\begin{tabular}{|c|c|c|c|c|c|c|c|c|c|c|}
\hline \multirow[b]{2}{*}{ Conditions } & \multicolumn{10}{|c|}{ Year of Onset } \\
\hline & 1991 & 1992 & 1993 & 1994 & 1995 & 1996 & 1997 & 1998 & 1999 & 2000 \\
\hline AIDS & 438 & 427 & 469 & 533 & 463 & 356 & 200 & 166 & 106 & 102 \\
\hline Adverse event after immunisation & 10 & 31 & 23 & 40 & 28 & 56 & 70 & 93 & 13 & 40 \\
\hline Total Arboviral* & 410 & 341 & 656 & 382 & 534 & 1226 & 1805 & 780 & 1217 & 964 \\
\hline Barmah Forest virus infections* & 6 & 6 & 25 & 40 & 271 & 172 & 187 & 134 & 249 & 189 \\
\hline Ross River virus infections* & 298 & 324 & 599 & 332 & 236 & 1032 & 1597 & 583 & 952 & 745 \\
\hline NOS $^{*}$ & 106 & 11 & 32 & 10 & 27 & 22 & 21 & 63 & 16 & 30 \\
\hline Blood lead level >=15ug/dl* & \multicolumn{5}{|c|}{ not notifiable until December 1996} & & 713 & 880 & 709 & 990 \\
\hline Botulism & 0 & 0 & 0 & 0 & 0 & 0 & 0 & 0 & 1 & 0 \\
\hline Brucellosis* & 2 & 2 & 4 & 4 & 2 & 1 & 3 & 3 & 2 & 1 \\
\hline Chancroid & \multicolumn{5}{|c|}{ not notifiable until December 1998} & & 0 & 0 & 1 & 0 \\
\hline Chlamydia trachomatis infections* & \multicolumn{5}{|c|}{ not notifiable until August 1998} & & 23 & 562 & 2438 & 3464 \\
\hline Cholera* & 1 & 0 & 1 & 0 & 1 & 3 & 1 & 1 & 2 & 0 \\
\hline Cryptosporidiosis* & \multicolumn{5}{|c|}{ not notifiable until December 1996} & & 157 & 1130 & 121 & 132 \\
\hline Food-borne illness(NOS) & 2744 & 253 & 106 & 213 & 270 & 211 & 255 & 201 & 151 & 125 \\
\hline Gastroenteritis (institutional) & 153 & 405 & 426 & 296 & 1359 & 554 & 939 & 737 & 635 & 640 \\
\hline Giardiasis* & \multicolumn{5}{|c|}{ not notifiable until August 1998} & 2 & 1 & 403 & 1091 & 968 \\
\hline Gonorrhoea* & 386 & 494 & 382 & 357 & 427 & 522 & 636 & 1048 & 1279 & 1048 \\
\hline Total H.influenzae & 211 & 219 & 124 & 61 & 29 & 13 & 17 & 11 & 13 & 8 \\
\hline H.influenzae type b epiglottitis* & 15 & 57 & 32 & 21 & 6 & 2 & 5 & 1 & 2 & 2 \\
\hline H.influenzae type b meningitis* & 47 & 104 & 53 & 17 & 11 & 4 & 3 & 3 & 3 & 1 \\
\hline H.influenzae type b septicaemia* & 11 & 26 & 24 & 12 & 8 & 3 & 1 & 4 & 6 & 4 \\
\hline H.influenzae type $b$ infection (NOS)* & 138 & 32 & 15 & 11 & 4 & 4 & 8 & 3 & 2 & 1 \\
\hline HIV infection* & 811 & 711 & 602 & 510 & 540 & 462 & 435 & 413 & 389 & 367 \\
\hline Haemolytic uraemic syndrome & \multicolumn{6}{|c|}{ not notifiable until December 1996} & 3 & 6 & 11 & 9 \\
\hline Hepatitis $A^{*}$ & 1120 & 903 & 579 & 586 & 615 & 958 & 1427 & 926 & 406 & 195 \\
\hline Hepatitis B: acute viral* & 412 & 115 & 96 & 75 & 63 & 43 & 52 & 53 & 63 & 94 \\
\hline Hepatitis B: other* & 1089 & 3131 & 3599 & 4033 & 4060 & 3549 & 3196 & 2988 & 3491 & 3914 \\
\hline Hepatitis C: acute viral ${ }^{*}$ & 22 & 28 & 23 & 22 & 33 & 19 & 19 & 102 & 82 & 138 \\
\hline Hepatitis C: other* & 828 & 3963 & 6006 & 7997 & 6983 & 7109 & 7043 & 7262 & 7630 & 7375 \\
\hline Hepatitis D* & 0 & 8 & 12 & 19 & 19 & 9 & 11 & 3 & 13 & 10 \\
\hline Hepatitis $E^{*}$ & 0 & 0 & 1 & 2 & 0 & 3 & 6 & 4 & 7 & 9 \\
\hline Total Legionnaires' disease & 37 & 104 & 66 & 60 & 75 & 74 & 33 & 46 & 41 & 41 \\
\hline L. longbeachae* & 0 & 14 & 13 & 8 & 16 & 30 & 9 & 19 & 12 & 12 \\
\hline L. pneumophila* & 16 & 80 & 34 & 30 & 35 & 34 & 18 & 22 & 22 & 26 \\
\hline NOS & 21 & 10 & 19 & 22 & 24 & 10 & 6 & 5 & 7 & 3 \\
\hline Leprosy & 1 & 7 & 5 & 3 & 3 & 2 & 0 & 0 & 1 & 2 \\
\hline Leptospirosis* & 28 & 21 & 16 & 14 & 6 & 33 & 33 & 50 & 55 & 53 \\
\hline Listeriosis ${ }^{*}$ & 11 & 13 & 12 & 10 & 14 & 22 & 23 & 28 & 22 & 18 \\
\hline Malaria* & 201 & 164 & 164 & 184 & 96 & 203 & 173 & 161 & 198 & 226 \\
\hline Total Measles & 493 & 808 & 2348 & 1484 & 596 & 191 & 273 & 119 & 32 & 32 \\
\hline Laboratory confirmed cases* & 20 & 76 & 460 & 302 & 138 & 35 & 98 & 19 & 13 & 20 \\
\hline other & 473 & 732 & 1888 & 1182 & 458 & 156 & 175 & 100 & 19 & 12 \\
\hline Total Meningococcal & 129 & 122 & 153 & 142 & 113 & 161 & 219 & 184 & 215 & 248 \\
\hline Meningitis & 54 & 94 & 98 & 80 & 72 & 99 & 109 & 56 & 109 & 105 \\
\hline Septicaemia & 16 & 19 & 44 & 41 & 27 & 41 & 67 & 77 & 71 & 78 \\
\hline NOS & 59 & 9 & 11 & 21 & 14 & 21 & 43 & 51 & 35 & 65 \\
\hline Mumps* & 8 & 23 & 13 & 11 & 14 & 27 & 29 & 39 & 32 & 92 \\
\hline Mycobacterial infection: other than TB* & 302 & 399 & 451 & 520 & 470 & 411 & 359 & 311 & 363 & 312 \\
\hline Paratyphoid* & 20 & 8 & 9 & 11 & 12 & 15 & 5 & 9 & 4 & 13 \\
\hline Pertussis & 50 & 217 & 1533 & 1408 & 1370 & 1157 & 4250 & 2311 & 1414 & 3682 \\
\hline Q Fever* & 166 & 213 & 404 & 267 & 202 & 287 & 258 & 236 & 164 & 128 \\
\hline Total Rubella* & 61 & 326 & 1186 & 233 & 2376 & 636 & 153 & 78 & 46 & 190 \\
\hline Rubella* & 60 & 326 & 1184 & 229 & 2375 & 631 & 153 & 78 & 45 & 190 \\
\hline Rubella (Congenital)* & 1 & 0 & 2 & 4 & 1 & 5 & 0 & 0 & 1 & 0 \\
\hline Total Salmonella* & 1172 & 805 & 980 & 1101 & 1366 & 1224 & 1698 & 1811 & 1423 & 1387 \\
\hline Salmonella bovis morbificans infections* & 19 & 21 & 32 & 24 & 15 & 13 & 25 & 40 & 22 & 39 \\
\hline Salmonella typhimurium infections* & 196 & 232 & 291 & 457 & 547 & 581 & 934 & 856 & 661 & 688 \\
\hline Salmonella infections (NOS) ${ }^{*}$ & 957 & 552 & 657 & 620 & 804 & 630 & 739 & 915 & 740 & 660 \\
\hline Total Syphilis & 581 & 877 & 739 & 976 & 833 & 663 & 513 & 598 & 522 & 535 \\
\hline Syphilis - >1 year duration* & 1 & 2 & 6 & 29 & 135 & 71 & 57 & 44 & 90 & 76 \\
\hline Syphilis - $<1$ year duration* & 1 & 5 & 7 & 22 & 31 & 38 & 26 & 22 & 100 & 154 \\
\hline Syphilis congenital & 1 & 1 & 0 & 2 & 7 & 3 & 4 & 1 & 3 & 1 \\
\hline Syphilis (NOS)* & 578 & 869 & 726 & 923 & 660 & 551 & 426 & 531 & 329 & 304 \\
\hline Tetanus & 5 & 2 & 5 & 4 & 0 & 1 & 3 & 3 & 1 & 2 \\
\hline Tuberculosis & 430 & 394 & 389 & 394 & 443 & 410 & 422 & 384 & 478 & 439 \\
\hline Typhoid* & 38 & 20 & 28 & 24 & 27 & 30 & 28 & 18 & 32 & 26 \\
\hline Verotoxin - producing E. coli infections* & & notifiak & until D & ember & & & 0 & 2 & 0 & 1 \\
\hline
\end{tabular}

* Laboratory-confirmed cases only $\quad$ NOS = Not Otherwise Specified

Following diseases have not been notified since 1991: Diphtheria*, Granuloma inguinale*, Lymphogranuloma venereum*, Plague*,

Poliomyelitis*, Rabies, Typhus*, Viral haemorrhagic fever, Yellow fever. 


\section{TABLE 2}

DISEASE NOTIFICATIONS BY PUBLIC HEALTH UNIT AREA, NSW, 2000

\begin{tabular}{|c|c|c|c|c|c|c|c|c|c|c|c|c|}
\hline \multirow[b]{3}{*}{ Conditions } & \multicolumn{10}{|c|}{ Health Area of residence } & & \\
\hline & \multicolumn{2}{|c|}{ CSA } & \multicolumn{2}{|c|}{ NSA } & \multicolumn{2}{|c|}{ WSA } & \multicolumn{2}{|c|}{ WEN } & \multicolumn{2}{|c|}{ SWS } & \multicolumn{2}{|c|}{ CCA } \\
\hline & No. & Rate & No. & Rate & No. & Rate & No. & Rate & No. & Rate & No. & Rate \\
\hline AIDS & 22 & 4.5 & 13 & 1.7 & 8 & 1.2 & 2 & 0.6 & 7 & 0.9 & 2 & 0.7 \\
\hline Adverse event after immunisation & 5 & 1.0 & 4 & 0.5 & 4 & 0.6 & 2 & 0.6 & 0 & 0.0 & 3 & 1.0 \\
\hline Total Arboviral* & 6 & 1.2 & 17 & 2.2 & 11 & 1.6 & 5 & 1.6 & 11 & 1.4 & 20 & 6.8 \\
\hline Barmah Forest virus infections* & 1 & 0.2 & 0 & 0.0 & 1 & 0.1 & 0 & 0.0 & 0 & 0.0 & 3 & 1.0 \\
\hline Ross River virus infections* & 2 & 0.4 & 10 & 1.3 & 9 & 1.3 & 5 & 1.6 & 3 & 0.4 & 17 & 5.8 \\
\hline NOS $^{*}$ & 3 & 0.6 & 7 & 0.9 & 1 & 0.1 & 0 & 0.0 & 8 & 1.0 & 0 & 0.0 \\
\hline Blood lead level >= 15ug/dl* & 32 & 6.5 & 23 & 3.0 & 100 & 14.6 & 30 & 9.5 & 143 & 18.2 & 5 & 1.7 \\
\hline Brucellosis* & 0 & 0.0 & 0 & 0.0 & 0 & 0.0 & 0 & 0.0 & 0 & 0.0 & 0 & 0.0 \\
\hline Chlamydia trachomatis infections* & 424 & 86.1 & 294 & 37.9 & 324 & 47.3 & 110 & 34.8 & 199 & 25.3 & 64 & 21.9 \\
\hline Cryptosporidiosis* & 5 & 1.0 & 3 & 0.4 & 2 & 0.3 & 6 & 1.9 & 2 & 0.3 & 0 & 0.0 \\
\hline Food-borne illness (NOS) & 34 & 6.9 & 4 & 0.5 & 3 & 0.4 & 0 & 0.0 & 5 & 0.6 & 1 & 0.3 \\
\hline Gastroenteritis (institutional) & 107 & 21.7 & 0 & 0.0 & 70 & 10.2 & 84 & 26.6 & 0 & 0.0 & 0 & 0.0 \\
\hline Giardiasis* & 55 & 11.2 & 121 & 15.6 & 99 & 14.4 & 45 & 14.2 & 74 & 9.4 & 40 & 13.7 \\
\hline Gonorrhoea* & 209 & 42.4 & 80 & 10.3 & 66 & 9.6 & 19 & 6.0 & 77 & 9.8 & 15 & 5.1 \\
\hline Total H.influenzae type b & 0 & 0.0 & 1 & 0.1 & 0 & 0.0 & 0 & 0.0 & 2 & 0.3 & 0 & 0.0 \\
\hline H.influenzae type b epiglottitis* & 0 & 0.0 & 1 & 0.1 & 0 & 0.0 & 0 & 0.0 & 0 & 0.0 & 0 & 0.0 \\
\hline H.influenzae type b meningitis* & 0 & 0.0 & 0 & 0.0 & 0 & 0.0 & 0 & 0.0 & 0 & 0.0 & 0 & 0.0 \\
\hline H.influenzae type b septicaemia* & 0 & 0.0 & 0 & 0.0 & 0 & 0.0 & 0 & 0.0 & 2 & 0.3 & 0 & 0.0 \\
\hline H.influenzae type b infection (NOS)* & 0 & 0.0 & 0 & 0.0 & 0 & 0.0 & 0 & 0.0 & 0 & 0.0 & 0 & 0.0 \\
\hline HIV infection* & 84 & 17.1 & 18 & 2.3 & 27 & 3.9 & 8 & 2.5 & 25 & 3.2 & 6 & 2.1 \\
\hline Haemolytic uraemic syndrome & 1 & 0.2 & 0 & 0.0 & 0 & 0.0 & 0 & 0.0 & 1 & 0.1 & 1 & 0.3 \\
\hline Hepatitis $A^{*}$ & 35 & 7.1 & 23 & 3.0 & 26 & 3.8 & 20 & 6.3 & 20 & 2.5 & 5 & 1.7 \\
\hline Hepatitis B: acute viral ${ }^{*}$ & 6 & 1.2 & 8 & 1.0 & 5 & 0.7 & 7 & 2.2 & 11 & 1.4 & 0 & 0.0 \\
\hline Hepatitis B: other* & 569 & 115.5 & 372 & 47.9 & 625 & 91.2 & 76 & 24.0 & 1362 & 173.5 & 35 & 12.0 \\
\hline Hepatitis C: acute viral ${ }^{*}$ & 12 & 2.4 & 32 & 4.1 & 0 & 0.0 & 17 & 5.4 & 1 & 0.1 & 1 & 0.3 \\
\hline Hepatitis C: other* & 772 & 156.7 & 338 & 43.6 & 1193 & 174.1 & 355 & 112.3 & 1012 & 128.9 & 326 & 111.5 \\
\hline Hepatitis D* & 0 & 0.0 & 0 & 0.0 & 1 & 0.1 & 0 & 0.0 & 4 & 0.5 & 0 & 0.0 \\
\hline Hepatitis $E^{*}$ & 1 & 0.2 & 1 & 0.1 & 2 & 0.3 & 1 & 0.3 & 1 & 0.1 & 0 & 0.0 \\
\hline Total Legionnaires' & 4 & 0.8 & 3 & 0.4 & 9 & 1.3 & 1 & 0.3 & 2 & 0.3 & 1 & 0.3 \\
\hline L.longbeachae* & 0 & 0.0 & 0 & 0.0 & 2 & 0.3 & 1 & 0.3 & 2 & 0.3 & 0 & 0.0 \\
\hline L.pneumophila* & 4 & 0.8 & 3 & 0.4 & 7 & 1.0 & 0 & 0.0 & 0 & 0.0 & 1 & 0.3 \\
\hline other & 0 & 0.0 & 0 & 0.0 & 0 & 0.0 & 0 & 0.0 & 0 & 0.0 & 0 & 0.0 \\
\hline Leprosy & 0 & 0.0 & 0 & 0.0 & 0 & 0.0 & 0 & 0.0 & 2 & 0.3 & 0 & 0.0 \\
\hline Leptospirosis* & 0 & 0.0 & 2 & 0.3 & 0 & 0.0 & 0 & 0.0 & 0 & 0.0 & 1 & 0.3 \\
\hline Listeriosis* & 2 & 0.4 & 1 & 0.1 & 1 & 0.1 & 1 & 0.3 & 3 & 0.4 & 1 & 0.3 \\
\hline Malaria* & 20 & 4.1 & 36 & 4.6 & 12 & 1.8 & 11 & 3.5 & 55 & 7.0 & 2 & 0.7 \\
\hline Total Measles & 4 & 0.8 & 6 & 0.8 & 2 & 0.3 & 2 & 0.6 & 5 & 0.6 & 1 & 0.3 \\
\hline Laboratory confirmed cases* & 2 & 0.4 & 6 & 0.8 & 1 & 0.1 & 1 & 0.3 & 3 & 0.4 & 1 & 0.3 \\
\hline other & 2 & 0.4 & 0 & 0.0 & 1 & 0.1 & 1 & 0.3 & 2 & 0.3 & 0 & 0.0 \\
\hline Total Meningococcal & 16 & 3.2 & 21 & 2.7 & 26 & 3.8 & 15 & 4.7 & 31 & 3.9 & 18 & 6.2 \\
\hline Meningitis & 10 & 2.0 & 8 & 1.0 & 13 & 1.9 & 7 & 2.2 & 12 & 1.5 & 4 & 1.4 \\
\hline Septicaemia & 5 & 1.0 & 10 & 1.3 & 13 & 1.9 & 8 & 2.5 & 11 & 1.4 & 1 & 0.3 \\
\hline NOS & 1 & 0.2 & 3 & 0.4 & 0 & 0.0 & 0 & 0.0 & 8 & 1.0 & 13 & 4.4 \\
\hline Mumps* & 12 & 2.4 & 11 & 1.4 & 23 & 3.4 & 3 & 0.9 & 17 & 2.2 & 2 & 0.7 \\
\hline Mycobacterial infection: other than TB* & 36 & 7.3 & 71 & 9.2 & 3 & 0.4 & 9 & 2.8 & 41 & 5.2 & 12 & 4.1 \\
\hline Paratyphoid* & 0 & 0.0 & 3 & 0.4 & 5 & 0.7 & 0 & 0.0 & 1 & 0.1 & 0 & 0.0 \\
\hline Pertussis & 109 & 22.1 & 298 & 38.4 & 337 & 49.2 & 175 & 55.4 & 262 & 33.4 & 120 & 41.1 \\
\hline Q Fever* & 1 & 0.2 & 1 & 0.1 & 1 & 0.1 & 1 & 0.3 & 2 & 0.3 & 1 & 0.3 \\
\hline Rubella* & 6 & 1.2 & 12 & 1.5 & 9 & 1.3 & 2 & 0.6 & 8 & 1.0 & 2 & 0.7 \\
\hline Total Salmonella* & 106 & 21.5 & 145 & 18.7 & 154 & 22.5 & 47 & 14.9 & 172 & 21.9 & 57 & 19.5 \\
\hline Salmonella bovis morbificans infections* & 5 & 1.0 & 3 & 0.4 & 3 & 0.4 & 3 & 0.9 & 7 & 0.9 & 3 & 1.0 \\
\hline Salmonella typhimurium infections* & 51 & 10.4 & 69 & 8.9 & 87 & 12.7 & 19 & 6.0 & 102 & 13.0 & 18 & 6.2 \\
\hline Salmonella infections (NOS) ${ }^{*}$ & 50 & 10.2 & 73 & 9.4 & 64 & 9.3 & 25 & 7.9 & 63 & 8.0 & 36 & 12.3 \\
\hline Total Syphilis & 80 & 16.2 & 27 & 3.5 & 59 & 8.6 & 8 & 2.5 & 101 & 12.9 & 9 & 3.1 \\
\hline Syphilis - <1 year duration* & 9 & 1.8 & 6 & 0.8 & 4 & 0.6 & 1 & 0.3 & 3 & 0.4 & 3 & 1.0 \\
\hline Syphilis - >1 year duration* & 42 & 8.5 & 11 & 1.4 & 14 & 2.0 & 3 & 0.9 & 10 & 1.3 & 4 & 1.4 \\
\hline Syphilis congenital & 0 & 0.0 & 0 & 0.0 & 0 & 0.0 & 0 & 0.0 & 0 & 0.0 & 0 & 0.0 \\
\hline Syphilis (NOS)* & 29 & 5.9 & 10 & 1.3 & 41 & 6.0 & 4 & 1.3 & 88 & 11.2 & 2 & 0.7 \\
\hline Tetanus & 0 & 0.0 & 0 & 0.0 & 0 & 0.0 & 0 & 0.0 & 0 & 0.0 & 0 & 0.0 \\
\hline Tuberculosis & 72 & 14.6 & 55 & 7.1 & 80 & 11.7 & 14 & 4.4 & 85 & 10.8 & 2 & 0.7 \\
\hline Typhoid* & 1 & 0.2 & 7 & 0.9 & 8 & 1.2 & 0 & 0.0 & 7 & 0.9 & 0 & 0.0 \\
\hline Verotoxin - producing E. coli infections* & 0 & 0.0 & 0 & 0.0 & 0 & 0.0 & 0 & 0.0 & 0 & 0.0 & 0 & 0.0 \\
\hline
\end{tabular}

* Laboratory-confirmed cases only $\quad$ NOS $=$ Not Otherwise Specified

Area health service population estimates 2000: CSA = Central Sydney Area (492 554); NSA = North Sydney Area (775 844); WSA = Western Sydney Area (685 350); WEN = Wentworth Area (316 064); SWS = South Western Sydney (785 124); CCA = Central Coast Area (292 303) 


\section{TABLE 2}

DISEASE NOTIFICATIONS BY PUBLIC HEALTH UNIT AREA, NSW, 2000 continued

\begin{tabular}{|c|c|c|c|c|c|c|c|c|c|c|c|c|}
\hline \multirow[b]{3}{*}{ Conditions } & \multicolumn{10}{|c|}{ Health Area of residence } & & \\
\hline & \multicolumn{2}{|c|}{ HUN } & \multicolumn{2}{|c|}{ ILL } & \multicolumn{2}{|c|}{ SES } & \multicolumn{2}{|c|}{ NRA } & \multicolumn{2}{|c|}{ MNC } & \multicolumn{2}{|c|}{ NEA } \\
\hline & No. & Rate & No. & Rate & No. & Rate & No. & Rate & No. & Rate & No. & Rate \\
\hline AIDS & 5 & 0.9 & 3 & 0.9 & 31 & 4.0 & 6 & 2.3 & 0 & 0.0 & 0 & 0.0 \\
\hline Adverse event after immunisation & 3 & 0.6 & 2 & 0.6 & 5 & 0.6 & 2 & 0.8 & 0 & 0.0 & 2 & 1.1 \\
\hline Total Arboviral* & 132 & 24.5 & 28 & 8.1 & 14 & 1.8 & 82 & 31.2 & 228 & 87.3 & 83 & 47.5 \\
\hline Barmah Forest virus infections* & 12 & 2.2 & 15 & 4.3 & 3 & 0.4 & 40 & 15.2 & 88 & 33.7 & 4 & 2.3 \\
\hline Ross River virus infections* & 119 & 22.1 & 13 & 3.7 & 9 & 1.2 & 39 & 14.8 & 140 & 53.6 & 79 & 45.2 \\
\hline NOS $^{*}$ & 1 & 0.2 & 0 & 0.0 & 2 & 0.3 & 3 & 1.1 & 0 & 0.0 & 0 & 0.0 \\
\hline Blood lead level >= 15ug/dl* & 441 & 81.9 & 18 & 5.2 & 36 & 4.7 & 11 & 4.2 & 7 & 2.7 & 4 & 2.3 \\
\hline Brucellosis* & 0 & 0.0 & 0 & 0.0 & 0 & 0.0 & 0 & 0.0 & 0 & 0.0 & 0 & 0.0 \\
\hline Chlamydia trachomatis infections* & 311 & 57.7 & 155 & 44.6 & 783 & 101.7 & 158 & 60.1 & 144 & 55.1 & 137 & 78.4 \\
\hline Cryptosporidiosis* & 8 & 1.5 & 4 & 1.2 & 12 & 1.6 & 26 & 9.9 & 11 & 4.2 & 23 & 13.2 \\
\hline Food-borne illness (NOS) & 2 & 0.4 & 0 & 0.0 & 55 & 7.1 & 0 & 0.0 & 2 & 0.8 & 0 & 0.0 \\
\hline Gastroenteritis (institutional) & 227 & 42.1 & 8 & 2.3 & 59 & 7.7 & 0 & 0.0 & 0 & 0.0 & 23 & 13.2 \\
\hline Giardiasis* & 62 & 11.5 & 26 & 7.5 & 140 & 18.2 & 136 & 51.8 & 32 & 12.2 & 40 & 22.9 \\
\hline Gonorrhoea* & 17 & 3.2 & 13 & 3.7 & 463 & 60.1 & 24 & 9.1 & 10 & 3.8 & 23 & 13.2 \\
\hline Total H.influenzae type b & 1 & 0.2 & 0 & 0.0 & 1 & 0.1 & 0 & 0.0 & 1 & 0.4 & 1 & 0.6 \\
\hline H.influenzae type b epiglottitis* & 0 & 0.0 & 0 & 0.0 & 0 & 0.0 & 0 & 0.0 & 1 & 0.4 & 0 & 0.0 \\
\hline H.influenzae type b meningitis* & 0 & 0.0 & 0 & 0.0 & 0 & 0.0 & 0 & 0.0 & 0 & 0.0 & 0 & 0.0 \\
\hline H.influenzae type b septicaemia* & 1 & 0.2 & 0 & 0.0 & 0 & 0.0 & 0 & 0.0 & 0 & 0.0 & 1 & 0.6 \\
\hline H.influenzae type b infection (NOS)* & 0 & 0.0 & 0 & 0.0 & 1 & 0.1 & 0 & 0.0 & 0 & 0.0 & 0 & 0.0 \\
\hline HIV infection* & 8 & 1.5 & 12 & 3.5 & 147 & 19.1 & 9 & 3.4 & 1 & 0.4 & 2 & 1.1 \\
\hline Haemolytic uraemic syndrome & 2 & 0.4 & 1 & 0.3 & 0 & 0.0 & 0 & 0.0 & 0 & 0.0 & 0 & 0.0 \\
\hline Hepatitis $A^{*}$ & 7 & 1.3 & 3 & 0.9 & 29 & 3.8 & 1 & 0.4 & 4 & 1.5 & 3 & 1.7 \\
\hline Hepatitis B: acute viral* & 9 & 1.7 & 4 & 1.2 & 23 & 3.0 & 8 & 3.0 & 2 & 0.8 & 4 & 2.3 \\
\hline Hepatitis B: other* & 63 & 11.7 & 71 & 20.4 & 516 & 67.0 & 21 & 8.0 & 16 & 6.1 & 39 & 22.3 \\
\hline Hepatitis C: acute viral* & 36 & 6.7 & 8 & 2.3 & 4 & 0.5 & 6 & 2.3 & 4 & 1.5 & 4 & 2.3 \\
\hline Hepatitis C: other* & 524 & 97.3 & 262 & 75.4 & 986 & 128.0 & 383 & 145.8 & 291 & 111.4 & 128 & 73.3 \\
\hline Hepatitis D* & 0 & 0.0 & 0 & 0.0 & 3 & 0.4 & 0 & 0.0 & 0 & 0.0 & 1 & 0.6 \\
\hline Hepatitis $E^{*}$ & 0 & 0.0 & 0 & 0.0 & 2 & 0.3 & 0 & 0.0 & 0 & 0.0 & 0 & 0.0 \\
\hline Total Legionnaires' & 2 & 0.4 & 6 & 1.7 & 4 & 0.5 & 0 & 0.0 & 1 & 0.4 & 0 & 0.0 \\
\hline L.longbeachae* & 0 & 0.0 & 1 & 0.3 & 1 & 0.1 & 0 & 0.0 & 0 & 0.0 & 0 & 0.0 \\
\hline L.pneumophila* & 0 & 0.0 & 4 & 1.2 & 3 & 0.4 & 0 & 0.0 & 1 & 0.4 & 0 & 0.0 \\
\hline other & 2 & 0.4 & 1 & 0.3 & 0 & 0.0 & 0 & 0.0 & 0 & 0.0 & 0 & 0.0 \\
\hline Leprosy & 0 & 0.0 & 0 & 0.0 & 0 & 0.0 & 0 & 0.0 & 0 & 0.0 & 0 & 0.0 \\
\hline Leptospirosis* & 9 & 1.7 & 0 & 0.0 & 0 & 0.0 & 13 & 4.9 & 5 & 1.9 & 16 & 9.2 \\
\hline Listeriosis* & 2 & 0.4 & 0 & 0.0 & 5 & 0.6 & 1 & 0.4 & 0 & 0.0 & 0 & 0.0 \\
\hline Malaria* & 12 & 2.2 & 7 & 2.0 & 33 & 4.3 & 7 & 2.7 & 9 & 3.4 & 8 & 4.6 \\
\hline Total Measles & 0 & 0.0 & 0 & 0.0 & 6 & 0.8 & 1 & 0.4 & 1 & 0.4 & 1 & 0.6 \\
\hline Measles : Laboratory confirmed cases* & 0 & 0.0 & 0 & 0.0 & 6 & 0.8 & 0 & 0.0 & 0 & 0.0 & 0 & 0.0 \\
\hline Measles : other & 0 & 0.0 & 0 & 0.0 & 0 & 0.0 & 1 & 0.4 & 1 & 0.4 & 1 & 0.6 \\
\hline Total Meningococcal & 13 & 2.4 & 26 & 7.5 & 37 & 4.8 & 6 & 2.3 & 5 & 1.9 & 4 & 2.3 \\
\hline Meningitis & 9 & 1.7 & 12 & 3.5 & 13 & 1.7 & 2 & 0.8 & 4 & 1.5 & 2 & 1.1 \\
\hline Septicaemia & 4 & 0.7 & 6 & 1.7 & 2 & 0.3 & 3 & 1.1 & 0 & 0.0 & 2 & 1.1 \\
\hline NOS & 0 & 0.0 & 8 & 2.3 & 22 & 2.9 & 1 & 0.4 & 1 & 0.4 & 0 & 0.0 \\
\hline Mumps* & 0 & 0.0 & 2 & 0.6 & 16 & 2.1 & 0 & 0.0 & 2 & 0.8 & 0 & 0.0 \\
\hline Mycobacterial infection: other than TB* & 26 & 4.8 & 12 & 3.5 & 31 & 4.0 & 10 & 3.8 & 25 & 9.6 & 5 & 2.9 \\
\hline Paratyphoid* & 0 & 0.0 & 0 & 0.0 & 3 & 0.4 & 1 & 0.4 & 0 & 0.0 & 0 & 0.0 \\
\hline Pertussis & 1043 & 193.6 & 100 & 28.8 & 264 & 34.3 & 82 & 31.2 & 100 & 38.3 & 138 & 79.0 \\
\hline Q Fever* & 6 & 1.1 & 3 & 0.9 & 0 & 0.0 & 37 & 14.1 & 20 & 7.7 & 9 & 5.2 \\
\hline Rubella* & 84 & 15.6 & 10 & 2.9 & 38 & 4.9 & 3 & 1.1 & 7 & 2.7 & 3 & 1.7 \\
\hline Total Salmonella* & 85 & 15.8 & 55 & 15.8 & 157 & 20.4 & 148 & 56.3 & 61 & 23.3 & 47 & 26.9 \\
\hline Salmonella bovis morbificans infections* & 3 & 0.6 & 2 & 0.6 & 3 & 0.4 & 0 & 0.0 & 1 & 0.4 & 0 & 0.0 \\
\hline Salmonella typhimurium infections* & 38 & 7.1 & 35 & 10.1 & 80 & 10.4 & 50 & 19.0 & 36 & 13.8 & 21 & 12.0 \\
\hline Salmonella infections (NOS)* & 44 & 8.2 & 18 & 5.2 & 74 & 9.6 & 98 & 37.3 & 24 & 9.2 & 26 & 14.9 \\
\hline Total Syphilis & 8 & 1.5 & 1 & 0.3 & 145 & 18.8 & 26 & 9.9 & 14 & 5.4 & 13 & 7.4 \\
\hline Syphilis - <1 year duration* & 2 & 0.4 & 0 & 0.0 & 12 & 1.6 & 5 & 1.9 & 5 & 1.9 & 10 & 5.7 \\
\hline Syphilis - >1 year duration* & 6 & 1.1 & 0 & 0.0 & 28 & 3.6 & 8 & 3.0 & 9 & 3.4 & 2 & 1.1 \\
\hline Syphilis congenital & 0 & 0.0 & 0 & 0.0 & 1 & 0.1 & 0 & 0.0 & 0 & 0.0 & 0 & 0.0 \\
\hline Syphilis (NOS)* & 0 & 0.0 & 1 & 0.3 & 104 & 13.5 & 13 & 4.9 & 0 & 0.0 & 1 & 0.6 \\
\hline Tetanus & 0 & 0.0 & 0 & 0.0 & 0 & 0.0 & 1 & 0.4 & 0 & 0.0 & 0 & 0.0 \\
\hline Tuberculosis & 11 & 2.0 & 8 & 2.3 & 86 & 11.2 & 3 & 1.1 & 12 & 4.6 & 4 & 2.3 \\
\hline Typhoid* & 0 & 0.0 & 0 & 0.0 & 2 & 0.3 & 0 & 0.0 & 0 & 0.0 & 1 & 0.6 \\
\hline Verotoxin - producing E. coli infections* & 0 & 0.0 & 0 & 0.0 & 0 & 0.0 & 0 & 0.0 & 0 & 0.0 & 0 & 0.0 \\
\hline
\end{tabular}

* lab-confirmed cases only NOS $=$ Not Otherwise Specified

Area health service population estimates 2000: HUN = Hunter Area (538 678); ILL = Illawarra Area (347 404); SES= South Eastern Sydney (770 097); NRA = Northern Rivers Area (262 774); MNC = Mid North Coast Area (261 316); NEA = New England Area (174 650) 


\section{TABLE 2}

DISEASE NOTIFICATIONS BY PUBLIC HEALTH UNIT AREA, NSW, 2000 continued

\begin{tabular}{|c|c|c|c|c|c|c|c|c|c|c|c|c|c|}
\hline \multirow[b]{3}{*}{ Conditions } & \multicolumn{11}{|c|}{ Health Area of residence } & & \\
\hline & \multicolumn{2}{|c|}{ MAC } & \multicolumn{2}{|c|}{ MWA } & \multicolumn{2}{|c|}{ FWA } & \multicolumn{2}{|c|}{ GMA } & \multicolumn{2}{|c|}{ SA } & \multirow{2}{*}{$\begin{array}{c}\text { NOS } \\
\text { No. }\end{array}$} & \multicolumn{2}{|c|}{ TOTAL } \\
\hline & No. & Rate & No. & Rate & No. & Rate & No. & Rate & No. & Rate & & No. & Rate \\
\hline AIDS & 0 & 0.0 & 0 & 0.0 & 0 & 0.0 & 1 & 0.4 & 2 & 1.1 & 0 & 102 & 1.6 \\
\hline Adverse event after immunisation & 1 & 1.0 & 0 & 0.0 & 2 & 4.1 & 4 & 1.6 & 1 & 0.5 & 0 & 40 & 0.6 \\
\hline Total Arboviral ${ }^{*}$ & 69 & 66.7 & 42 & 25.1 & 69 & 142.3 & 121 & 46.9 & 26 & 14.1 & 0 & 964 & 14.9 \\
\hline Barmah Forest virus infections* & 3 & 2.9 & 2 & 1.2 & 3 & 6.2 & 4 & 1.6 & 10 & 5.4 & 0 & 189 & 2.9 \\
\hline Ross River virus infections* & 66 & 63.8 & 40 & 23.9 & 64 & 132.0 & 114 & 44.2 & 16 & 8.7 & 0 & 745 & 11.5 \\
\hline NOS $^{*}$ & 0 & 0.0 & 0 & 0.0 & 2 & 4.1 & 3 & 1.2 & 0 & 0.0 & 0 & 30 & 0.5 \\
\hline Blood lead level >= 15ug/dl* & 12 & 11.6 & 1 & 0.6 & 112 & 231.0 & 6 & 2.3 & 5 & 2.7 & 4 & 990 & 15.3 \\
\hline Brucellosis* & 1 & 1.0 & 0 & 0.0 & 0 & 0.0 & 0 & 0.0 & 0 & 0.0 & 0 & 1 & 0.0 \\
\hline Chlamydia trachomatis infections* & 48 & 46.4 & 74 & 44.2 & 66 & 136.1 & 103 & 39.9 & 63 & 34.2 & 7 & 3464 & 53.6 \\
\hline Cryptosporidiosis* & 7 & 6.8 & 1 & 0.6 & 1 & 2.1 & 12 & 4.7 & 9 & 4.9 & 0 & 132 & 2.0 \\
\hline Food-borne illness (NOS) & 19 & 18.4 & 0 & 0.0 & 0 & 0.0 & 0 & 0.0 & 0 & 0.0 & 0 & 125 & 1.9 \\
\hline Gastroenteritis (institutional) & 0 & 0.0 & 62 & 37.1 & 0 & 0.0 & 0 & 0.0 & 0 & 0.0 & 0 & 640 & 9.9 \\
\hline Giardiasis* & 35 & 33.8 & 21 & 12.6 & 5 & 10.3 & 24 & 9.3 & 12 & 6.5 & 1 & 968 & 15.0 \\
\hline Gonorrhoea* & 4 & 3.9 & 11 & 6.6 & 9 & 18.6 & 1 & 0.4 & 4 & 2.2 & 3 & 1048 & 16.2 \\
\hline Total H.influenzae type b & 0 & 0.0 & 0 & 0.0 & 0 & 0.0 & 1 & 0.4 & 0 & 0.0 & 0 & 8 & 0.1 \\
\hline H.influenzae type b epiglottitis* & 0 & 0.0 & 0 & 0.0 & 0 & 0.0 & 0 & 0.0 & 0 & 0.0 & 0 & 2 & 0.0 \\
\hline H.influenzae type b meningitis* & 0 & 0.0 & 0 & 0.0 & 0 & 0.0 & 1 & 0.4 & 0 & 0.0 & 0 & 1 & 0.0 \\
\hline H.influenzae type b septicaemia* & 0 & 0.0 & 0 & 0.0 & 0 & 0.0 & 0 & 0.0 & 0 & 0.0 & 0 & 4 & 0.1 \\
\hline H.influenzae type b infection (NOS)* & 0 & 0.0 & 0 & 0.0 & 0 & 0.0 & 0 & 0.0 & 0 & 0.0 & 0 & 1 & 0.0 \\
\hline HIV infection* & 1 & 1.0 & 5 & 3.0 & 0 & 0.0 & 2 & 0.8 & 1 & 0.5 & 11 & 367 & 5.7 \\
\hline Haemolytic uraemic syndrome & 0 & 0.0 & 2 & 1.2 & 1 & 2.1 & 0 & 0.0 & 0 & 0.0 & 0 & 9 & 0.1 \\
\hline Hepatitis $A^{*}$ & 2 & 1.9 & 13 & 7.8 & 1 & 2.1 & 1 & 0.4 & 2 & 1.1 & 0 & 195 & 3.0 \\
\hline Hepatitis B: acute viral ${ }^{*}$ & 1 & 1.0 & 1 & 0.6 & 0 & 0.0 & 4 & 1.6 & 1 & 0.5 & 0 & 94 & 1.5 \\
\hline Hepatitis B: other* & 9 & 8.7 & 16 & 9.6 & 48 & 99.0 & 30 & 11.6 & 32 & 17.4 & 14 & 3914 & 60.6 \\
\hline Hepatitis C: acute viral ${ }^{*}$ & 1 & 1.0 & 9 & 5.4 & 0 & 0.0 & 3 & 1.2 & 0 & 0.0 & 0 & 138 & 2.1 \\
\hline Hepatitis C: other* & 68 & 65.7 & 278 & 166.2 & 45 & 92.8 & 195 & 75.6 & 191 & 103.8 & 28 & 7375 & 114.1 \\
\hline Hepatitis D* & 0 & 0.0 & 1 & 0.6 & 0 & 0.0 & 0 & 0.0 & 0 & 0.0 & 0 & 10 & 0.2 \\
\hline Hepatitis $E^{*}$ & 0 & 0.0 & 0 & 0.0 & 0 & 0.0 & 0 & 0.0 & 1 & 0.5 & 0 & 9 & 0.1 \\
\hline Total Legionnaires' & 1 & 1.0 & 4 & 2.4 & 0 & 0.0 & 1 & 0.4 & 2 & 1.1 & 0 & 41 & 0.6 \\
\hline L.longbeachae* & 0 & 0.0 & 3 & 1.8 & 0 & 0.0 & 1 & 0.4 & 1 & 0.5 & 0 & 12 & 0.2 \\
\hline L.pneumophila* & 1 & 1.0 & 1 & 0.6 & 0 & 0.0 & 0 & 0.0 & 1 & 0.5 & 0 & 26 & 0.4 \\
\hline other & 0 & 0.0 & 0 & 0.0 & 0 & 0.0 & 0 & 0.0 & 0 & 0.0 & 0 & 3 & 0.0 \\
\hline Leprosy & 0 & 0.0 & 0 & 0.0 & 0 & 0.0 & 0 & 0.0 & 0 & 0.0 & 0 & 2 & 0.0 \\
\hline Leptospirosis* & 1 & 1.0 & 3 & 1.8 & 0 & 0.0 & 2 & 0.8 & 1 & 0.5 & 0 & 53 & 0.8 \\
\hline Listeriosis* & 0 & 0.0 & 1 & 0.6 & 0 & 0.0 & 0 & 0.0 & 0 & 0.0 & 0 & 18 & 0.3 \\
\hline Malaria* & 0 & 0.0 & 4 & 2.4 & 1 & 2.1 & 4 & 1.6 & 5 & 2.7 & 0 & 226 & 3.5 \\
\hline Total Measles & 2 & 1.9 & 0 & 0.0 & 1 & 2.1 & 0 & 0.0 & 0 & 0.0 & 0 & 32 & 0.5 \\
\hline Laboratory confirmed cases* & 0 & 0.0 & 0 & 0.0 & 0 & 0.0 & 0 & 0.0 & 0 & 0.0 & 0 & 20 & 0.3 \\
\hline other & 2 & 1.9 & 0 & 0.0 & 1 & 2.1 & 0 & 0.0 & 0 & 0.0 & 0 & 12 & 0.2 \\
\hline Total Meningococcal & 7 & 6.8 & 6 & 3.6 & 2 & 4.1 & 9 & 3.5 & 5 & 2.7 & 1 & 248 & 3.8 \\
\hline Meningitis & 2 & 1.9 & 2 & 1.2 & 1 & 2.1 & 3 & 1.2 & 0 & 0.0 & 1 & 105 & 1.6 \\
\hline Septicaemia & 1 & 1.0 & 3 & 1.8 & 1 & 2.1 & 5 & 1.9 & 3 & 1.6 & 0 & 78 & 1.2 \\
\hline NOS & 4 & 3.9 & 1 & 0.6 & 0 & 0.0 & 1 & 0.4 & 2 & 1.1 & 0 & 65 & 1.0 \\
\hline Mumps* & 1 & 1.0 & 0 & 0.0 & 1 & 2.1 & 1 & 0.4 & 0 & 0.0 & 1 & 92 & 1.4 \\
\hline Mycobacterial infection: other than TB* & 1 & 1.0 & 10 & 6.0 & 0 & 0.0 & 16 & 6.2 & 4 & 2.2 & 0 & 312 & 4.8 \\
\hline Paratyphoid* & 0 & 0.0 & 0 & 0.0 & 0 & 0.0 & 0 & 0.0 & 0 & 0.0 & 0 & 13 & 0.2 \\
\hline Pertussis & 108 & 104.3 & 234 & 139.9 & 2 & 4.1 & 199 & 77.1 & 111 & 60.3 & 0 & 3682 & 57.0 \\
\hline Q Fever* & 21 & 20.3 & 12 & 7.2 & 8 & 16.5 & 1 & 0.4 & 4 & 2.2 & 0 & 128 & 2.0 \\
\hline Rubella* & 0 & 0.0 & 1 & 0.6 & 0 & 0.0 & 2 & 0.8 & 3 & 1.6 & 0 & 190 & 2.9 \\
\hline Total Salmonella* & 22 & 21.3 & 29 & 17.3 & 6 & 12.4 & 64 & 24.8 & 29 & 15.8 & 3 & 1387 & 21.5 \\
\hline Salmonella bovis morbificans infections* & 0 & 0.0 & 2 & 1.2 & 0 & 0.0 & 2 & 0.8 & 1 & 0.5 & 1 & 39 & 0.6 \\
\hline Salmonella typhimurium infections ${ }^{*}$ & 13 & 12.6 & 9 & 5.4 & 0 & 0.0 & 41 & 15.9 & 19 & 10.3 & 0 & 688 & 10.6 \\
\hline Salmonella infections (NOS)* & 9 & 8.7 & 18 & 10.8 & 6 & 12.4 & 21 & 8.1 & 9 & 4.9 & 2 & 660 & 10.2 \\
\hline Total Syphilis & 9 & 8.7 & 15 & 9.0 & 14 & 28.9 & 3 & 1.2 & 1 & 0.5 & 2 & 535 & 8.3 \\
\hline Syphilis - <1 year duration* & 4 & 3.9 & 1 & 0.6 & 11 & 22.7 & 0 & 0.0 & 0 & 0.0 & 0 & 76 & 1.2 \\
\hline Syphilis - >1 year duration* & 1 & 1.0 & 9 & 5.4 & 3 & 6.2 & 3 & 1.2 & 1 & 0.5 & 0 & 154 & 2.4 \\
\hline Syphilis congenital & 0 & 0.0 & 0 & 0.0 & 0 & 0.0 & 0 & 0.0 & 0 & 0.0 & 0 & 1 & 0.0 \\
\hline Syphilis (NOS)* & 4 & 3.9 & 5 & 3.0 & 0 & 0.0 & 0 & 0.0 & 0 & 0.0 & 2 & 304 & 4.7 \\
\hline Tetanus & 0 & 0.0 & 0 & 0.0 & 1 & 2.1 & 0 & 0.0 & 0 & 0.0 & 0 & 2 & 0.0 \\
\hline Tuberculosis & 2 & 1.9 & 3 & 1.8 & 2 & 4.1 & 0 & 0.0 & 0 & 0.0 & 0 & 439 & 6.8 \\
\hline Typhoid* & 0 & 0.0 & 0 & 0.0 & 0 & 0.0 & 0 & 0.0 & 0 & 0.0 & 0 & 26 & 0.4 \\
\hline Verotoxin - producing E. coli infections* & 0 & 0.0 & 0 & 0.0 & 0 & 0.0 & 1 & 0.4 & 0 & 0.0 & 0 & 1 & 0.0 \\
\hline
\end{tabular}

* lab-confirmed cases only NOS $=$ Not Otherwise Specified

Area health service population estimates 2000: MAC = Macquarie Area (103 506); MWA = Mid Western Area (167 262) FWA =

Far West Area (48 478); GMA = Greater Murray Area (257 940); SA = Southern Area (184 082); TOTAL = Total population in NSW (6 463 426) 


\section{TABLE 3}

\section{DISEASE NOTIFICATIONS, BY AGE AND SEX, NSW, 2000}

\begin{tabular}{|c|c|c|c|c|c|c|c|c|c|c|c|c|c|}
\hline \multirow{2}{*}{$\begin{array}{l}0-4 \text { yrs } \\
\text { Conditions }\end{array}$} & \multicolumn{2}{|c|}{$5-24$ yrs } & \multicolumn{2}{|c|}{$25-44$ yrs } & \multicolumn{2}{|c|}{$45-64$ yrs } & \multicolumn{2}{|c|}{$>=65$ yrs } & \multicolumn{2}{|c|}{ Total } & \multirow[b]{2}{*}{ M } & \multirow[b]{2}{*}{$\mathbf{F}$} & \multirow[b]{2}{*}{$\mathbf{U} / \mathbf{T}$} \\
\hline & M & $\mathbf{F}$ & $\mathbf{M}$ & $\mathbf{F}$ & $\mathbf{M}$ & $\mathbf{F}$ & $\mathbf{M}$ & $\mathbf{F}$ & $\mathbf{M}$ & $\mathbf{F}$ & & & \\
\hline AIDS & 0 & 0 & 0 & 1 & 59 & 11 & 25 & 2 & 4 & 0 & 88 & 14 & 0 \\
\hline Adverse event after immunisation & 17 & 11 & 0 & 4 & 0 & 2 & 1 & 2 & 1 & 1 & 19 & 20 & 1 \\
\hline Total Arboviral* & 1 & 1 & 71 & 56 & 236 & 188 & 183 & 140 & 52 & 35 & 543 & 420 & 1 \\
\hline Barmah Forest virus infections* & 1 & 0 & 10 & 4 & 36 & 30 & 47 & 30 & 15 & 15 & 109 & 79 & 1 \\
\hline Ross River virus infections* & 0 & 1 & 58 & 51 & 190 & 155 & 128 & 107 & 35 & 20 & 411 & 334 & 0 \\
\hline $\mathrm{NOS}^{*}$ & 0 & 0 & 3 & 1 & 10 & 3 & 8 & 3 & 2 & 0 & 23 & 7 & 0 \\
\hline Blood lead level >= 15ug/dl $(a)^{*}$ & 37 & 20 & 124 & 8 & 511 & 14 & 238 & 12 & 24 & 0 & 934 & 54 & 2 \\
\hline Brucellosis* & 0 & 0 & 0 & 0 & 0 & 0 & 1 & 0 & 0 & 0 & 1 & 0 & 0 \\
\hline Chlamydia trachomatis infections* (a) & 13 & 12 & 480 & 1176 & 974 & 619 & 110 & 48 & 10 & 3 & 1590 & 1860 & 14 \\
\hline Cryptosporidiosis* & 48 & 19 & 13 & 16 & 16 & 9 & 8 & 2 & 0 & 1 & 85 & 47 & 0 \\
\hline Food-borne illness (NOS) & 5 & 0 & 15 & 16 & 31 & 25 & 15 & 12 & 2 & 4 & 68 & 57 & 0 \\
\hline Gastroenteritis (institutional) & 67 & 56 & 9 & 27 & 14 & 65 & 10 & 63 & 67 & 260 & 167 & 473 & 0 \\
\hline Giardiasis* & 168 & 116 & 109 & 87 & 173 & 142 & 78 & 59 & 14 & 22 & 542 & 426 & 0 \\
\hline Gonorrhoea* & 1 & 1 & 161 & 42 & 691 & 37 & 101 & 5 & 8 & 1 & 962 & 86 & 0 \\
\hline Total H.influenzae type b & 3 & 1 & 0 & 0 & 0 & 1 & 1 & 0 & 0 & 2 & 4 & 4 & 0 \\
\hline H.influenzae type b epiglottitis* & 0 & 0 & 0 & 0 & 0 & 1 & 0 & 0 & 0 & 1 & 0 & 2 & 0 \\
\hline H.influenzae type b meningitis* & 1 & 0 & 0 & 0 & 0 & 0 & 0 & 0 & 0 & 0 & 1 & 0 & 0 \\
\hline H.influenzae type b septicaemia* & 1 & 1 & 0 & 0 & 0 & 0 & 1 & 0 & 0 & 1 & 2 & 2 & 0 \\
\hline H.influenzae type b infection (NOS)* & 1 & 0 & 0 & 0 & 0 & 0 & 0 & 0 & 0 & 0 & 1 & 0 & 0 \\
\hline HIV infection* & 1 & 1 & 23 & 6 & 223 & 20 & 64 & 2 & 5 & 0 & 325 & 30 & 12 \\
\hline Haemolytic uraemic syndrome & 3 & 0 & 0 & 2 & 1 & 0 & 0 & 1 & 1 & 1 & 5 & 4 & 0 \\
\hline Hepatitis $A^{*}$ & 6 & 0 & 39 & 29 & 67 & 18 & 16 & 10 & 5 & 5 & 133 & 62 & 0 \\
\hline Hepatitis B: acute viral ${ }^{*}$ & 0 & 0 & 20 & 25 & 26 & 10 & 9 & 1 & 3 & 0 & 58 & 36 & 0 \\
\hline Hepatitis B: other* & 10 & 11 & 349 & 334 & 1211 & 997 & 520 & 294 & 86 & 81 & 2176 & 1717 & 21 \\
\hline Hepatitis C: acute viral ${ }^{*}$ & 0 & 0 & 27 & 33 & 46 & 24 & 5 & 3 & 0 & 0 & 78 & 60 & 0 \\
\hline Hepatitis C: other* & 29 & 14 & 898 & 585 & 3059 & 1505 & 712 & 314 & 125 & 110 & 4824 & 2529 & 22 \\
\hline Hepatitis D* & 0 & 0 & 1 & 1 & 5 & 2 & 1 & 0 & 0 & 0 & 7 & 3 & 0 \\
\hline Hepatitis $E^{*}$ & 0 & 0 & 4 & 3 & 0 & 2 & 0 & 0 & 0 & 0 & 4 & 5 & 0 \\
\hline Total Legionnaires' & 0 & 1 & 1 & 0 & 2 & 2 & 15 & 3 & 13 & 4 & 31 & 10 & 0 \\
\hline L.longbeachae* & 0 & 0 & 0 & 0 & 0 & 0 & 6 & 2 & 4 & 0 & 10 & 2 & 0 \\
\hline L.pneumophila* & 0 & 0 & 1 & 0 & 2 & 1 & 9 & 1 & 9 & 3 & 21 & 5 & 0 \\
\hline other & 0 & 1 & 0 & 0 & 0 & 1 & 0 & 0 & 0 & 1 & 0 & 3 & 0 \\
\hline Leprosy & 0 & 0 & 0 & 0 & 1 & 1 & 0 & 0 & 0 & 0 & 1 & 1 & 0 \\
\hline Leptospirosis* & 0 & 0 & 6 & 3 & 19 & 4 & 16 & 2 & 3 & 0 & 44 & 9 & 0 \\
\hline Listeriosis* & 0 & 1 & 0 & 0 & 0 & 0 & 4 & 1 & 6 & 6 & 10 & 8 & 0 \\
\hline Malaria* & 1 & 4 & 54 & 11 & 96 & 27 & 21 & 6 & 5 & 1 & 177 & 49 & 0 \\
\hline Total Measles & 8 & 6 & 5 & 10 & 2 & 1 & 0 & 0 & 0 & 0 & 15 & 17 & 0 \\
\hline Measles : Laboratory confirmed cases* & * 3 & 4 & 4 & 6 & 2 & 1 & 0 & 0 & 0 & 0 & 9 & 11 & 0 \\
\hline Measles : other & 5 & 2 & 1 & 4 & 0 & 0 & 0 & 0 & 0 & 0 & 6 & 6 & 0 \\
\hline Total Meningococcal & 39 & 35 & 72 & 46 & 20 & 13 & 7 & 12 & 0 & 4 & 138 & 110 & 0 \\
\hline Meningitis & 13 & 13 & 34 & 25 & 3 & 4 & 3 & 8 & 0 & 2 & 53 & 52 & 0 \\
\hline Septicaemia & 17 & 14 & 20 & 10 & 7 & 4 & 2 & 2 & 0 & 2 & 46 & 32 & 0 \\
\hline NOS & 9 & 8 & 18 & 11 & 10 & 5 & 2 & 2 & 0 & 0 & 39 & 26 & 0 \\
\hline Mumps* & 1 & 0 & 28 & 29 & 15 & 13 & 3 & 3 & 0 & 0 & 47 & 45 & 0 \\
\hline Mycobacterial infection: other than TB* & 7 & 6 & 5 & 4 & 42 & 19 & 49 & 34 & 71 & 75 & 174 & 138 & 0 \\
\hline Paratyphoid* & 0 & 0 & 3 & 2 & 4 & 1 & 1 & 2 & 0 & 0 & 8 & 5 & 0 \\
\hline Pertussis & 128 & 154 & 856 & 883 & 318 & 535 & 259 & 370 & 82 & 97 & 1643 & 2039 & 0 \\
\hline Q Fever* & 2 & 0 & 21 & 4 & 36 & 16 & 31 & 11 & 5 & 2 & 95 & 33 & 0 \\
\hline Rubella* & 2 & 2 & 112 & 20 & 34 & 12 & 3 & 3 & 2 & 0 & 153 & 37 & 0 \\
\hline Total Salmonella* & 223 & 217 & 213 & 195 & 145 & 158 & 61 & 72 & 45 & 52 & 687 & 694 & 6 \\
\hline Bovis morbificans infections* & 9 & 8 & 6 & 3 & 3 & 5 & 2 & 1 & 1 & 1 & 21 & 18 & 0 \\
\hline Typhimurium infections* & 118 & 110 & 126 & 119 & 58 & 67 & 18 & 26 & 18 & 25 & 338 & 347 & 3 \\
\hline NOS* & 96 & 99 & 81 & 73 & 84 & 86 & 41 & 45 & 26 & 26 & 328 & 329 & 3 \\
\hline Total Syphilis & 1 & 1 & 19 & 42 & 113 & 120 & 124 & 23 & 55 & 35 & 313 & 221 & 1 \\
\hline Syphilis - <1 year duration* & 0 & 0 & 5 & 15 & 24 & 18 & 10 & 2 & 1 & 1 & 40 & 36 & 0 \\
\hline Syphilis - >1 year duration* & 1 & 0 & 9 & 9 & 30 & 43 & 27 & 8 & 17 & 10 & 84 & 70 & 0 \\
\hline Syphilis congenital & 0 & 1 & 0 & 0 & 0 & 0 & 0 & 0 & 0 & 0 & 0 & 1 & 0 \\
\hline Syphilis (NOS)* & 0 & 0 & 5 & 18 & 59 & 59 & 87 & 13 & 37 & 24 & 189 & 114 & 1 \\
\hline Tetanus & 0 & 0 & 0 & 0 & 0 & 0 & 0 & 0 & 0 & 2 & 0 & 2 & 0 \\
\hline Tuberculosis & 5 & 9 & 20 & 31 & 83 & 86 & 56 & 48 & 55 & 46 & 219 & 220 & 0 \\
\hline Typhoid* & 1 & 1 & 9 & 6 & 4 & 3 & 1 & 1 & 0 & 0 & 15 & 11 & 0 \\
\hline Verotoxin - producing E. coli infections* & 0 & 1 & 0 & 0 & 0 & 0 & 0 & 0 & 0 & 0 & 0 & 1 & 0 \\
\hline
\end{tabular}

* Laboratory-confirmed cases only $\quad$ NOS $=$ Not Otherwise Specified

(a) $=(1+1)$ trans sexual case 


\section{TABLE 4}

DISEASE NOTIFICATIONS BY MONTH OF ONSET, NSW, 2000

Conditions

Months of Onset

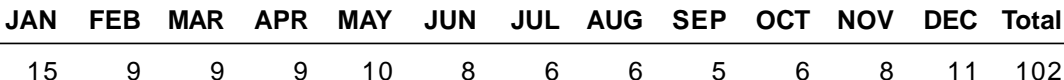

AIDS

Adverse event after immunisation

Total Arboviral*

Barmah Forest virus infections*

Ross River virus infections*

NOS*

Blood lead level >=15ug/dl*

Brucellosis*

Chlamydia trachomatis infections*

Cryptosporidiosis*

Food-borne illness(NOS)

Gastroenteritis (institutional)

Giardiasis*

Gonorrhoea*

Total H.influenzae*

H.influenzae type b epiglottitis*

$H$.influenzae type b meningitis*

$\mathrm{H}$.influenzae type b septicaemia

$\mathrm{H}$.influenzae type b infection (NOS)*

HIV infection*

Haemolytic uraemic syndrome

Hepatitis $A^{*}$

Hepatitis B: acute viral ${ }^{*}$

Hepatitis B: other*

Hepatitis C: acute viral ${ }^{\star}$

Hepatitis C: other*

Hepatitis D*

Hepatitis $E^{*}$

Total Legionnaires'

L. longbeachae*

L. pneumophila*

NOS

Leprosy

Leptospirosis*

Listeriosis*

Malaria*

Total Measles

Measles : Laboratory confirmed cases

Measles : other

Total Meningococcal

Meningitis

Septicaemia

NOS

Mumps*

Mycobacterial infection: other than TB*

Paratyphoid*

Pertussis

Q Fever*

Rubella*

Total Salmonella*

Salmonella bovis morbificans infections*

Salmonella typhimurium infections*

Salmonella infections (NOS)*

Total Syphilis

Syphilis - $<1$ year duration*

Syphilis - $>1$ year duration*

Syphilis congenital

Syphilis (NOS)*

Tetanus

Tuberculosis

Typhoid*

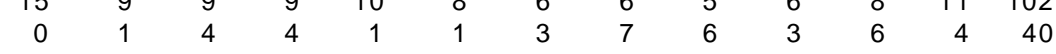

$\begin{array}{rrrrrrrrrrrrr}64 & 113 & 132 & 185 & 204 & 67 & 32 & 26 & 26 & 30 & 45 & 40 & 964\end{array}$

$\begin{array}{lllllllllllll}16 & 25 & 17 & 15 & 30 & 14 & 8 & 11 & 17 & 10 & 17 & 9 & 189\end{array}$

$\begin{array}{lllllllllllll}42 & 83 & 110 & 162 & 174 & 51 & 24 & 14 & 8 & 20 & 28 & 29 & 745\end{array}$

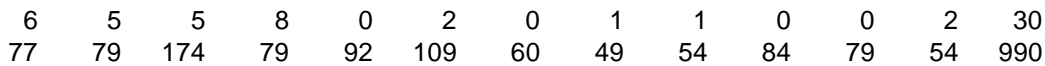

$\begin{array}{rrrrrrrrrrrrr}0 & 0 & 1 & 0 & 0 & 0 & 0 & 0 & 0 & 0 & 0 & 0 & 1\end{array}$

$\begin{array}{lllllllllllll}207 & 211 & 254 & 213 & 222 & 283 & 287 & 383 & 283 & 378 & 415 & 328 & 3464\end{array}$

$\begin{array}{lllllllllllll}12 & 14 & 17 & 13 & 7 & 7 & 10 & 3 & 8 & 14 & 18 & 9 & 132\end{array}$

$\begin{array}{lllllllllllll}4 & 29 & 2 & 0 & 31 & 0 & 34 & 0 & 0 & 9 & 5 & 11 & 125\end{array}$

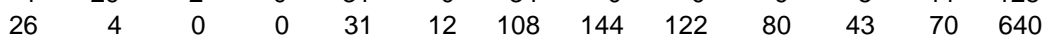

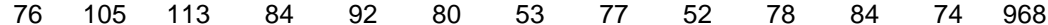

$\begin{array}{lllllllllllll}103 & 95 & 111 & 83 & 98 & 84 & 77 & 68 & 93 & 76 & 85 & 75 & 1048\end{array}$

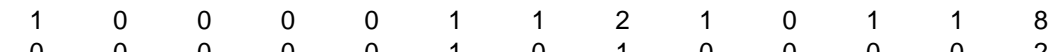

$\begin{array}{llllllllllll}1 & 0 & 0 & 0 & 0 & 0 & 0 & 0 & 0 & 0 & 0 & 0\end{array}$

$\begin{array}{rrrrrrrrrrrrr}0 & 0 & 0 & 0 & 0 & 0 & 1 & 1 & 1 & 0 & 0 & 1 & 4 \\ 0 & 0 & 0 & 0 & 0 & 0 & 0 & 0 & 0 & 0 & 1 & 0 & 1\end{array}$

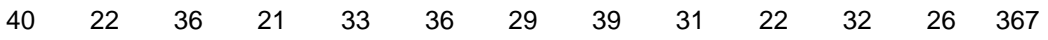

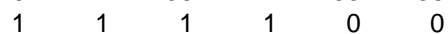

$\begin{array}{llllll}29 & 16 & 19 & 16 & 13 & 18\end{array}$

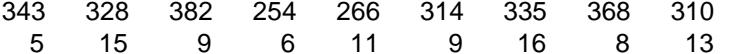

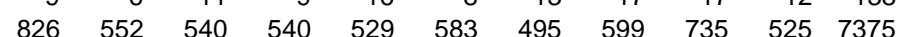

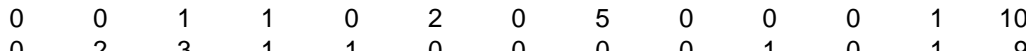

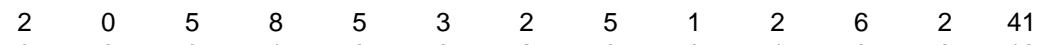

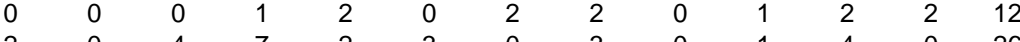

$\begin{array}{rrrrrrrrrrrrr}0 & 0 & 1 & 0 & 1 & 0 & 0 & 0 & 1 & 0 & 0 & 0 & 3\end{array}$

$\begin{array}{rrrrrrrrrrrr}0 & 0 & 0 & 0 & 1 & 0 & 0 & 0 & 0 & 0 & 1 & 0\end{array}$

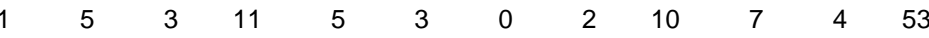

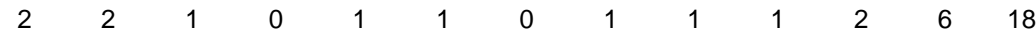

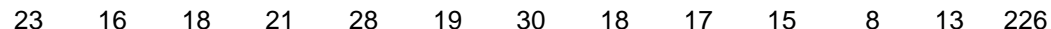

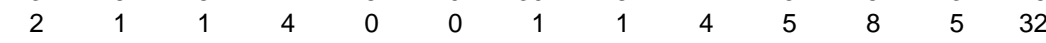

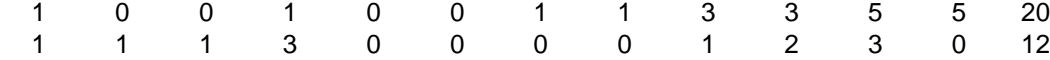

$\begin{array}{lllllllllllll}19 & 10 & 11 & 15 & 14 & 29 & 27 & 29 & 33 & 20 & 20 & 21 & 248\end{array}$

$\begin{array}{rrrrrrrrrrrr}7 & 3 & 4 & 7 & 4 & 8 & 4 & 6 & 13 & 8 & 10 & 4\end{array}$

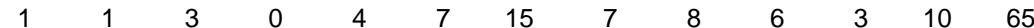

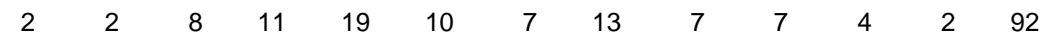

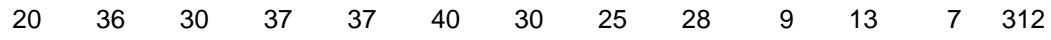

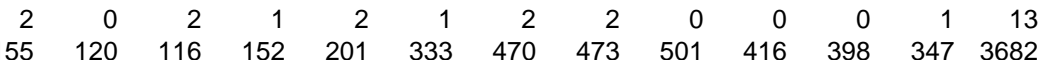

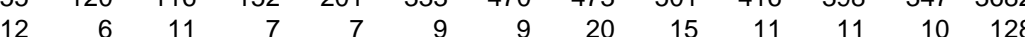

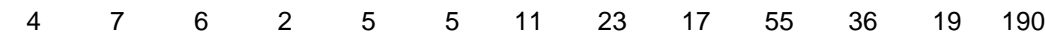

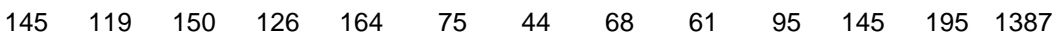

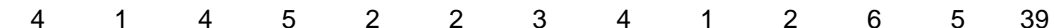

$\begin{array}{lllllllllllll}76 & 48 & 75 & 70 & 90 & 38 & 24 & 25 & 31 & 36 & 61 & 114 & 688\end{array}$

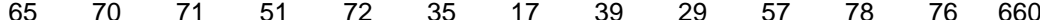

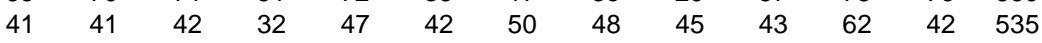

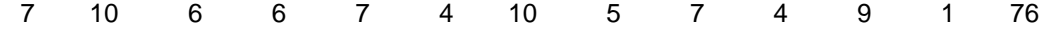

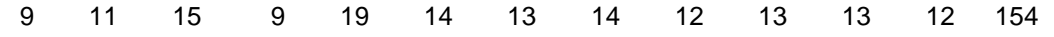

$\begin{array}{rrrrrrrrrrrrr}0 & 0 & 0 & 0 & 1 & 0 & 0 & 0 & 0 & 0 & 0 & 0 & 1\end{array}$

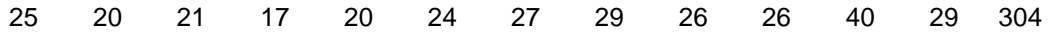

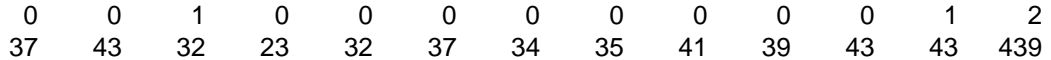

Verotoxin - producing E. coli infections*

* Laboratory-confirmed cases only $\quad \mathrm{NOS}=$ Not Otherwise Specified 